\title{
Décisions médicales relatives à la fin de vie
}

\author{
Le thème des décisions médicales en fin de vie va être examiné par l'Institut de méde- \\ cine sociale et préventive de l'Université de Zurich et par l'Institut d'éthique biomédi- \\ cale de l'Université de Genève lors d'une étude réalisée en collaboration à ce sujet. \\ L'équipe de recherche demande au corps médical de la soutenir dans cet important \\ projet et de prendre le temps de répondre au questionnaire, qui sera adressé per- \\ sonnellement. Nous souhaitons vous informer sur les enjeux de cette étude et la ma- \\ nière dont elle se déroulera.
}

\begin{abstract}
Margareta Schmida,
Ueli Zellweger ${ }^{a}$,

Matthias Bopp ${ }^{a}$,

Georg Bosshard,

Karin Faisst ${ }^{a}$,

Samia Hurst ${ }^{b}$,

Milo Puhan ${ }^{a}$,

Felix Gutzwiller ${ }^{a}$

a Institut de médecine sociale et préventive de l'Université de Zurich

b Institut d'éthique biomédicale de l'Université de Genève

c Clinique de gériatrie Hôpital universitaire de Zurich et

Les médecins doivent souvent prendre de difficiles décisions éthiques. Lorsque la fin de la vie est proche, il faut parfois prendre des décisions pour ou contre un traitement médical, qui va influencer sur la durée finale de la vie du patient. Pour la Suisse alémanique, cela a été nettement démontré [1-3] par l'étude EURELD* en 2001 déjà. Il est à présent important de faire le point de la situation actuelle dans la pratique médicale, et de le faire cette fois à l'échelle de toute la Suisse. Nous avons en effet vu dans l'intervalle des modifications des conditions-cadres, une évolution sociale accélérée, comme par ex. l'explosion démographique, le nouveau droit de la protection de la personne, la création accrue d'instituts spécialisés tels que centres de soins palliatifs.
\end{abstract} Zentrum Alter und Mobilität, Universität Zürich und Stadtspital Waid

* EURELD (Medical End-of-Life
Decisions in 6 European Countries)

Correspondance:

Institut de médecine sociale et préventive de l'Université de Zurich

Dr Margareta Schmid Hirschengraben 84 CH-8001 Zurich

schmid[at]access.uzh.ch

\section{Décisions médicales relatives à la «fin de vie»}

L'Institut de médecine sociale et préventive de l'Université de Zurich mènera en collaboration avec l'Institut d'éthique biomédicale de l'Université de Genève entre août 2013 et janvier 2014 une étude intitulée «Décisions médicales relatives à la fin de vie: fréquence et tendances en Suisse» [4], comme projet partiel du programme national de recherche (PNR) 67 «Fin de vie» [5]. Cette étude devrait permettre de récolter des informations fiables sur la prise de décisions dans la pratique réelle. La nouvelle étude englobe - contrairement à celle de 2001 - toutes les parties du pays, de sorte qu'elle va permettre de faire des comparaisons entre les différentes régions linguistiques. Les questions centrales du questionnaire sont inchangées par rapport à l'étude de 2001 et sont formulées dans une forme également identique à celles d'études menées dans ce domaine en Hollande et en Belgique (ainsi qu'en France avec certaines restrictions). C'est pourquoi cette nouvelle étude va permettre une mise en perspective non seulement nationale mais également internationale, ainsi qu'une comparaison avec les résultats de l'étude 2001.
L'étude est soutenue par l'Académie Suisse des Sciences Médicales et par la FMH. L'Office féd. de la statistique (OFS) apporte également son importante contribution dans la réalisation du projet.

\section{Une étude sur la base des cas de décès}

L'OFS effectuera chaque semaine, dès août 2013 et pour une période de 6 mois, une sélection aléatoire des certifications de décès annoncés par les offices d'état civil. Il sera tenu compte de décès d'individus âgés d' un an ou plus. Au total, 9000 décès seront explorés par un questionnaire remis dans toute la Suisse aux médecins traitants (Suisse alémanique 5000, Suisse romande 3000, Suisse italienne 1000). Un échantillon de cette ampleur est nécessaire pour pouvoir se faire une idée fondée sur les décisions.

Le questionnaire tient sur quatre pages et englobe avec ses 33 questions les thèmes suivants: pour ou contre quelles mesures le médecin a-t-il décidé? Quel était le but de ces décisions? Qui a été impliqué dans le processus de la prise de décision? Il faut au maximum une quinzaine de minutes pour remplir le questionnaire. Lorsqu'il s'agit d'un décès subit et inattendu, le questionnaire est encore plus rapidement rempli avec moins de questions, qui demandent peu de temps pour répondre. Il concerne un cas de décès, qui dans 90 pourcent des cas a eu lieu moins de 3 semaines auparavant.

Du fait que l'échantillonnage est basé sur un pointage aléatoire des cas de décès et non pas sur un choix au hasard des médecins, il est possible que certains d'entre vous soient inscrits plusieurs fois pour participer à cette enquête. Les résultats d'un sondage ont montré que les deux tiers de tous les médecins inclus sont appelés à remplir le questionnaire une seule fois, $25 \%$ deux ou trois fois et environ $10 \%$ plus de trois fois. Afin que les résultats soient représentatifs pour toute la population, il est important de ne pas s'arrê- 
ter après le premier questionnaire, mais de remplir également les autres le cas échéant.

\section{Protection des données}

L'enquête est entièrement indépendante des informations en lien avec les causes du décès, qui sont automatiquement enregistrées par l'OFS auprès des médecins. Les réponses données dans le cadre de cette étude ne pourront jamais être mises en lien avec la statistique des causes de décès, ni lors de l'étude ni à l'avenir. L'OFS fournit par contre à l'avance certaines informations d'ordre démographique concernant la personne décédée à l'Institut de médecine sociale et préventive de l'Université de Zurich.

L'étude est conçue de manière à préserver l'anonymat des données à tous les échelons et en tout temps. Dès lors que chaque questionnaire est pourvu d'un code individuel, l'on a opté pour un procédé de sauvegarde de l'anonymat à quatre niveaux:

- Le questionnaire rempli est envoyé à l'Académie suisse des sciences médicales (ASSM). Il ne renferme aucune information qui permette à quiconque d'identifier le médecin traitant, ni la personne décédée.

- L'ASSM indique au fur et à mesure à l'Institut de médecine sociale et préventive le code chiffré de chacun des questionnaires qui lui sont remis.

- L'Institut de médecine sociale et préventive efface de sa banque de données chaque code, nom et adresse du médecin, numéro d'enregistrement du

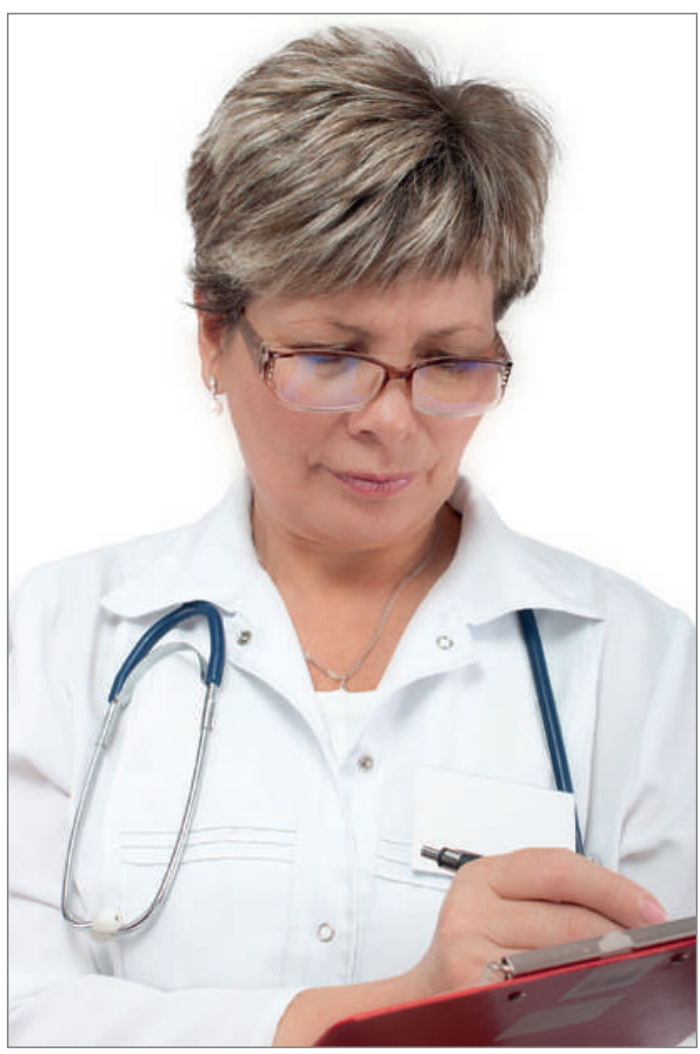

Pour que cet éclairage donne une image exacte de la réalité, il est important que le plus possible de médecins participent. décès ainsi que les dates de la naissance et du décès. Dès lors, il n'y a plus aucune possibilité pour personne de retrouver d'une manière ou d'une autre le médecin traitant ni la personne décédée. - C'est seulement dans un deuxième temps que l'Académie transmet à l'Institut de médecine sociale et préventive les questionnaires pour leur analyse.

La Commission cantonale d'éthique du canton de Zurich a examiné l'étude et atteste que sa conception et son déroulement sont irréprochables du point de vue de l'éthique (KEK-StV-No 23/13). Le chargé de la protection des données du canton de Zurich confirme que lors de la vérification des exigences relatives à la protection des données, il a été reconnu que les mesures et conditions prévues sont remplies.

\section{Remarque finale}

Lors de la prise de décision dans les cas de fin de vie, différents critères interviennent. Parmi eux, les expériences, émotions, croyances et appréciations des valeurs de la part des médecins, d'autres professionnels de la santé, des patients et de leurs proches. Cette étude vise à fournir des bases scientifiques, ainsi qu'un éclairage rationnel et objectif, aux thèmes difficiles qui accompagnent les décisions médicales en fin de vie. Pour que cet éclairage donne une image exacte de la réalité, il est bien sûr important que le plus possible de médecins soient contactés pour participer à l'enquête et que le plus possible de questionnaires soient dûment remplis et renvoyés. Cela avait été le cas il y 12 ans: à tel point que la participation et la qualité des indications ont été considérées en Suisse comme excellentes dans la comparaison internationale.

L'équipe de recherche remercie d'ores et déjà tous les participants pour leur contribution à la réussite de cette étude.

\section{Références}

1 van der Heide A, Deliens L, Faisst K, Nilstun T, Norup M, Paci E, et al. End-of-life decision-making in 6 European Countries. Descriptive Study. Lancet. 2003;362:345-50.

2 Bosshard G, Faisst K, Fischer S, Minder R, Zellweger U, Tschopp A, et al. Begrenzung lebenserhaltender Massnahmen bei Patienten am Lebensende in der deutschsprachigen Schweiz - Resultate einer Todesfallstudie. Dtsch Med Wochenschr. 2005;130:1-6.

3 Faisst K, Fischer S, Bosshard G, Zellweger U, Bär W, Gutzwiller F. Décisions médicales en fin de vie dans six pays européens: Premiers résultats. Projet européen «Medical End-of-Life Decisions: Attitudes and Practices in 6 European Countries». Bull Méd Suisses. 2003;84(34):1730-2.

4 www.nfp67.ch/F/projets/decisions-motifs-attitudes/ decisions-medicales-fin-vie-frequence-tendancessuisse/Pages/default.aspx

5 www.nfp67.ch/F/Pages/home.aspx 